\section{Multi-slice CT Urography after diuretic injection: initial results diuretic injection: initial results}

\begin{abstract}
Multidetektor $\boldsymbol{C T}$ nach Injektion eines Diuretikums: Erste Ergebnisse. Ziel: Untersuchung zur Durchführbarkeit der CT Urographie (CTU) mit einem Multidetektor(MD)-Computertomographen und ob eine niedrigdosierte Injektion eines Diuretikums für die Kontrastierung des Harntraktes vorteilhaft ist. Methode: Bei 21 Patienten mit urologischen Erkrankungen wurde eine MD-CTU durchgeführt. 5/21 Pat. erhielten $250 \mathrm{ml}$ physiologische $\mathrm{NaCl}$-Lösung i.v. Bei $16 / 21$ Pat. wurden $10 \mathrm{mg}$ Furosemid 3-5 min vor Kontrastmittelgabe injiziert. Es wurden eine $4 \times 2,5 \mathrm{~mm}$ Kollimation, ein Pitch von 1,25 und eine Stromstärke von $100-150 \mathrm{~mA}$ verwendet. Die Messzeit betrug 12$16 \mathrm{~s}$. Es wurden $3 \mathrm{~mm}$ dünne axiale Schichten mit einer Überlappung von $67 \%$ rekonstruiert. Für die urographische Darstellung wurden multiplanare Maximum-Intensitäts-Projektionen (MIP) berechnet. Die Elimination von überlagernden Knochen erfolgte mit Hilfe der Volume-Of-Interest Methode. Ergebnisse: Die Furosemid-verstärkte MD-CTU ermöglichte eine nahezu vollständige oder komplette Kontrastierung von 30 der 32 (94\%) Ureteren und von allen 32 (100\%) Nierenbeckenkelchsystemen bis zu einem Serumkreatinin von $150 \mu \mathrm{mol} / \mathrm{l}$. Es war jeweils nur ein CTU-Scan bei unseren Patienten erforderlich. Eine externe Harnleiterkompression wurde nicht benötigt. Bei Gabe von physiologischer $\mathrm{NaCl}$-Lösung waren die Dichtewerte im Nierenbecken inhomogener und 4-5 mal höher als bei Verwendung eines Diuretikums. In Kombination mit Furosemid war eine genauere Darstellung von Kelchdetails möglich und Konkremente ließen sich innerhalb des kontrastierten Urins gut identifizieren und von Phlebolithen sicher unterscheiden. Nachteilig waren Nachverarbeitungszeiten von bis zu $20 \mathrm{~min}$. Kontrastierte Darmschlingen konnten auf MIP-Bildern zu Überlagerungen führen. Schlussfolgerungen: Erste Resultate zeigen eine gute Durchführbarkeit der Furosemid-verstärkten MD-CTU zur detaillierten Darstellung des oberen Harntraktes.
\end{abstract}

Schlüsselwörter: Urographie - Computertomographie - Multidetektor-Technologie - Furosemid

Purpose: To investigate the feasibility of CT urography (CTU) using a multi-slice (MS) scanner and to find out whether a lowdose diuretic injection is advantageous for the opacification of the urinary tract. Methods: MS-CTU was performed in 21 patients with urologic diseases. In 5/21 patients, $250 \mathrm{ml}$ of physiologic saline Solution were injected. In $16 / 21$ patients, $10 \mathrm{mg}$ of

Fortschr Röntgenstr 2001; 173: 176 - 180

(c) Georg Thieme Verlag Stuttgart · New York

ISSN 1438-9029
C. C. A. Nolte-Ernsting ${ }^{1}$, J. E. Wildberger ${ }^{1}$, H. Borchers ${ }^{2}$,

T. Schmitz-Rode ${ }^{1}$, R. W. Günther ${ }^{1}$

${ }^{1}$ Departments of Diagnostic Radiology

2 Urology

University of Technology, Aachen

furosemide were injected $3-5$ min before contrast material administration. A $4 \times 2.5 \mathrm{~mm}$ collimation with a pitch of 1.25 and a tube curent of $100-150 \mathrm{~mA}$ were used. Scan time was $12-16 \mathrm{sec} .3 \mathrm{~mm}$ thin axial images with an overlap of $67 \%$ were reconstructed. Multiplanar maximum intensity projection (MIP) images were postprocessed to obtain urographic views. Bone structures were eliminated using the volume-of-interest method. Results: Furosemide-enhanced MS-CTU achieved either near complete or complete opacification in 30/32 (94\%) ureters and in $32 / 32(100 \%)$ pelvicaliceal systems up to a serum creatinine of $150 \mu \mathrm{mol} / \mathrm{I}$. In our series, only one CTU scan per patient was needed to obtain a diagnostic urogram after 10 min of contrast material injection. Ureteral compression was not necessary. When physiologic saline solution was used instead of furosemide, the radiopacity inside the enhanced pelvicalices was 4 - 5 times higher and more inhomogeneous. Diuretic-enhanced MS-CTU was more accurate in the depiction of pelvicaliceal details. In combination with furosemide, calculi were well identified inside the opacified urine and were safely differentiated from phleboliths. Postprocessing times of up to 20 minutes were problematic as were contrast-enhanced superimposing bowel loops on MIP images. Conclusion: Preliminary results demonstrate a good feasibility of furosemide-enhanced MS-CTU for obtaining detailed visualization of the entire upper urinary tract.

Key words: Urography - Computed tomography - Multi-slice technology - Furosemide

\section{Introduction}

In modern uroradiology, unenhanded helical CT meanwhile became established as the best imaging modality for the diagnosis of urolithiasis [1]. In contrast, urographic visualization of the urinary tract anatomy by means of contrast materialenhanced CT in the excretory phase could not really compete with intravenous pyelography (IVP), although several studies demonstrated a general feasibility of CT urography (CTU) [2,3]. In comparison with IVP, the conventional CTU examination technique still revealed a deficiency in the accurate delineation of pelvicaliceal details [3]. Furthermore, complete opacification of all ureteral segments seemed to be an inconsistent finding in 
previous CT urograms which necessitated the use of a compression device even in CT [3]. In the present context, we assume that two aspects can help to improve the examination technique for CT urography: Firstly, it makes sense to utilize all the technical advantages of the new multi-slice technology [4] also for the performance of CTU. Secondly, derived from the knowledge of the positive interaction of low-dose furosemide and contrast material for optimizing the morphologic accuracy in excretory MR urography [5], it would be interesting to know whether this modification can also be successfully applied to CT urography. Therefore, the purpose of the preliminary study was to obtain initial results as to whether diuretic-enhanced multislice CT urography (MS-CTU) can improve the quality of routine $\mathrm{CT}$ to visualize the urinary tract in the excretory phase.

\section{Patients and methods}

Multi-slice CT urography was performed in 21 patients ( 12 men, 9 women), ranging in age from 38 to 72 years. The serum creatinine level was normal in 18 patients. Two patients had an increased serum creatinine of $1.5 \mathrm{mg} / \mathrm{dl}(133 \mu \mathrm{mol} / \mathrm{l})$ and one patient of $1.7 \mathrm{mg} / \mathrm{dl}(150 \mu \mathrm{mol} / \mathrm{l})$. Twenty patients were referred to our department for a multiphase CT examination of the kidneys and urinary tracts because of suspected extrinsic or intrinsic tumor disease by sonography $(n=9)$, by intravenous pyelography $(n=1)$, or by CT from an external department $(n=1)$ and, moreover, because of sonographically diagnosed hydronephrosis $(n=1)$, hematuria and/or flank pain of uncertain cause $(n=7)$, and hematuria after trauma $(n=1)$. In another patient with a carcinoma of the cervix, MS-CTU was carried out to provide a preoperative assessment of the ureters. Informed consent was obtained from each patient after the procedure of the examination had been explained carefully, including the additional injection of low-dose furosemide (Lasix; Hoechst, Germany). No extra CT scans were scheduled for the multiphase examination protocol, which included the excretory phase.

All CT examinations were performed using a multi-slice scanner (Somatom Volume Zoom; Siemens, Forchheim, Germany). The patients were placed in supine position. For contrast-enhancement, all patients received a single intravenous bolus injection of $100 \mathrm{ml}$ of Iopromide (Ultravist 370; Schering, Berlin, Germany) at a flow rate of $3 \mathrm{ml} / \mathrm{sec}$. In 16 of 21 patients, $10 \mathrm{mg}$ of furosemide were intravenously administered 3 - 5 minutes before contrast material injection, followed by a bolus of $30 \mathrm{ml}$ of physiologic saline solution immediately after application of contrast material. In 5 of 21 patients, MS-CTU was performed without use of furosemide, but with intravenous bolus injection of $250 \mathrm{ml}$ of physiologic saline solution $(150 \mathrm{ml} 5 \mathrm{~min}$ before, $100 \mathrm{ml}$ immediately after contrast material injection). The contrast agent and the saline solution were administered via a standard double power-injector (Liebel-Flarsheim, Cincinatti, U.S.A.). Oral contrast material opacification of bowel loops was applied in only one patient.

After completion of the standard CT scans in the corticomedullary and nephrographic phases, a low-dose single CT slice „test-image“ (20mAs) was obtained from the kidneys within 10 minutes after contrast material injection in order to make sure that the contrast agent had definitely reached the pelvicaliceal system on either side. With the information of this „testimage“, it was possible to start the subsequent CT urography scan exactly in the excretory phase. In each case, the CTU scan was planned to extend from the upper pole of the left kidney down to the symphysis. An abdominal compression device was not used. The following scan parameters were employed for MS-CTU: 4 x $2.5 \mathrm{~mm}$ collimation; $12.5 \mathrm{~mm}$ table feed per rotation; pitch $1.25 ; 120 \mathrm{kV} ; 100$ - $150 \mathrm{mAs} ; 0.5 \mathrm{sec}$ rotation time; standard abdominal kernel. All data were acquired within a short breath-hold lasting between 12 and 16 sec encompassing a z-coverage of up to $40 \mathrm{~cm}$. For the diagnostic reading, axial images were reconstructed with a slice thickness and increment of $5 \mathrm{~mm}$. For postprocesing procedures, we reconstructed source images with a slice thickness of $3 \mathrm{~mm}$ and an increment of $1 \mathrm{~mm}$ generating an overlap of $67 \%$. From these source images, maximum intensity projection (MIP) images in multiple different planes were reconstructed using the standard software (Wizard, Siemens) of the CT scanner. In addition, superimposing bones and calcified arteries were eliminated from the MIP images using the volume of interest (VOI) software tool also implemented on our system.

The MS-CT urograms were analyzed by two radiologists (author 1 and 2) in a consensus assessment concerning the feasibilty of the new technique, the completeness of urinary tract visualization, and pathologic findings. The ability of MS-CTU to visualize the upper urinary tratcs was evaluated separately for the ureters and pelvicaliceal systems on either side using a scale from 1 to 3 , with

1. fragmented opacification of the ureter/pelvicalices with distinct gaps in the contrast material column.

2. near complete visualization of the ureter/pelvicalices with only one or two short unopacified segments; (a) with limited caliceal detail and (b) with good caliceal detail.

3. complete opacification of the ureter/pelvicalices; (a) with limited caliceal detail and (b) with good caliceal detail.

The assessment comprised a total of 42 pelvicaliceal systems and ureters. The urinary bladder was not included in this preliminary analysis. For a quantitative assessment, the Hounsfield units (HU) of each contrast material-enhanced renal pelvis were measured in the axial CTU source images using the region-of-interest (ROI) method.

\section{Results}

In the five patients (10 pelvicalices and ureters) in whom physiologic saline solution was administered instead of lowdose furosemide, complete opacification of the collecting system was possible, however, the scores varied markedly between the different patients (Tab.1). With use of saline solution, density values between 947 and $2544 \mathrm{HU}$ (mean: 1514 $\mathrm{HU}$ ) were measured inside the 10 opacified pelvicaliceal systems after 10 min of contrast material injection. A mean standard deviation of $352 \mathrm{HU}$ (23\%) per ROI was indicative of a relatively inhomogeneous bright enhancement. The demonstration of pelvicaliceal details was assessed as limited in all cases (Tab.1). In MS-CTU performed using saline solution, the calices frequently appeared to be blunted and blurred (Fig. 1). Dense contrast material inside the papillae and medullary collecting tubules partly also affected the sharp delineation of the caliceal fornices.

In the 16 patients ( 32 pelvicalices and ureters) who underwent diuretic-enhanced MS-CTU, the injection of $10 \mathrm{mg}$ of furose- 
Table 1 Consensus assessment of two reviewers: scores for the visualization of the upper urinary tract in MS-CTU.

\begin{tabular}{|c|c|c|c|c|}
\hline score & $\begin{array}{l}\text { MS-CTU } \\
\text { ureter } \\
(n=10)\end{array}$ & $\begin{array}{l}\mathrm{VaCl} 0.9 \% \\
\text { pelvicalices } \\
(\mathrm{n}=10)\end{array}$ & $\begin{array}{l}\text { MS-CTU w } \\
\text { ureter } \\
(n=32)\end{array}$ & $\begin{array}{l}\text { h furosemide } \\
\text { pelvicalices } \\
(\mathrm{n}=32)\end{array}$ \\
\hline 1 & $4(40 \%)$ & $1(10 \%)$ & $2(6 \%)$ & 0 \\
\hline 2 & $3(30 \%)$ & $\begin{array}{l}\text { a } 2(20 \%) \\
\text { b } 0\end{array}$ & $8(25 \%)$ & $\begin{array}{lll}\text { a } & 2 & (6 \%) \\
\text { b } & 2 & (6 \%)\end{array}$ \\
\hline 3 & $3(30 \%)$ & $\begin{array}{l}\text { a } 7(70 \%) \\
\text { b } 0\end{array}$ & 22 (69\%) & $\begin{array}{l}\text { a } 1 \quad(3 \%) \\
\text { b } 27(85 \%)\end{array}$ \\
\hline
\end{tabular}

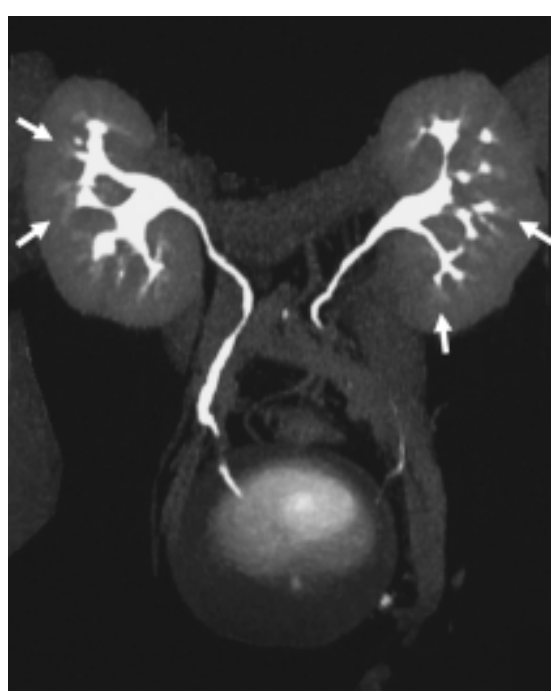

Fig. 1 MIP image from MS-CTU performed after injection of $250 \mathrm{ml}$ physiologic saline solution instead of furosemide in a 53year old man. Although the pelvicaliceal systems are brightly enhanced, the calices appear blunted. Dense contrast material is also seen in many papillae (arrows).

mide led to uniform distribution of the subsequently excreted contrast material inside the urinary tracts. In each of our patients, only one test-scan and one breath-hold MS-CTU scan were necessary to provide a sufficient urographic view after 10 minutes following contrast material injection. A good urographic effect was possible even in those three patients with an increased serum creatinine of up to $1.7 \mathrm{mg} / \mathrm{dl}(150 \mu \mathrm{mol} / \mathrm{l})$. Near complete or complete opacification was constantly achieved with use of low-dose furosemide (Tab.1). In two cases, the left ureter was insufficiently visualized. In one of these patients, the left ureter was superimposed by an inserted radiopaque double-J stent. Artefacts caused by very dense endoluminal accumulation of contrast material were not observed. Morphologic details such as the caliceal fornices were frequently visualized (Fig. 2). After injection of furosemide, the density values measured inside the 32 opacified pelvicalices were markedly lower than obtained without a diuretic agent, now ranging between 181 and $492 \mathrm{HU}$ (mean: $354 \mathrm{HU}$ ). A low mean standard deviation of $21 \mathrm{HU}(6 \%)$ per ROI indicated a very uniform endoluminal opacification.

The postprocessing time per patient lasted up to 20 minutes. Most of this time had to be spent for the elimination of skeletal structures using the VOI method. Multiplanar MIP images enabled to assess each calyx from any preferred view without superposition of neighbouring calices. The distal segments of the ureters were best seperated from the bladder in the near lateral MIP images (Fig. $\mathbf{2}$ b, 3). Orally administered contrast material inside of bowel loops partially affected the visibility of

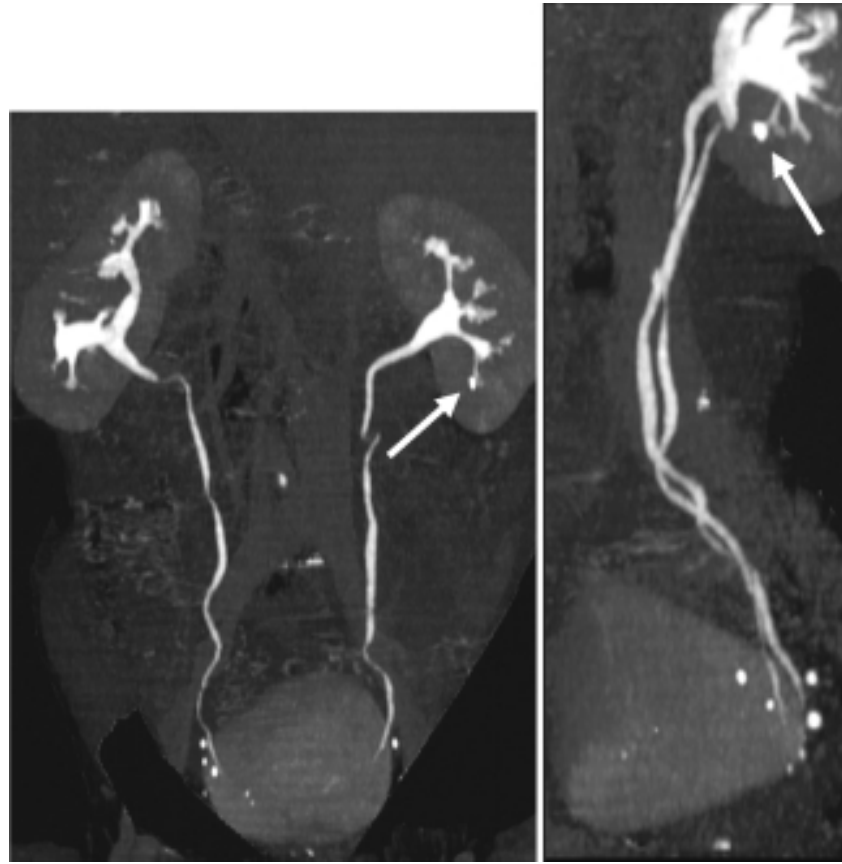

Fig. 2 MIP images from furosemide-enhanced MS-CTU in a 45-year old male patient. Complete opacification of the tortuously running ureters and sharp delineation of the pelvicaliceal systems are achieved 10 min after injection of contrast material. The anterior (a) and lateral (b) MIP images demonstrate a calculus inside a lower pole calix (arrow) of the left kidney, whereas phleboliths are clearly separated from the distal ureteral segments.

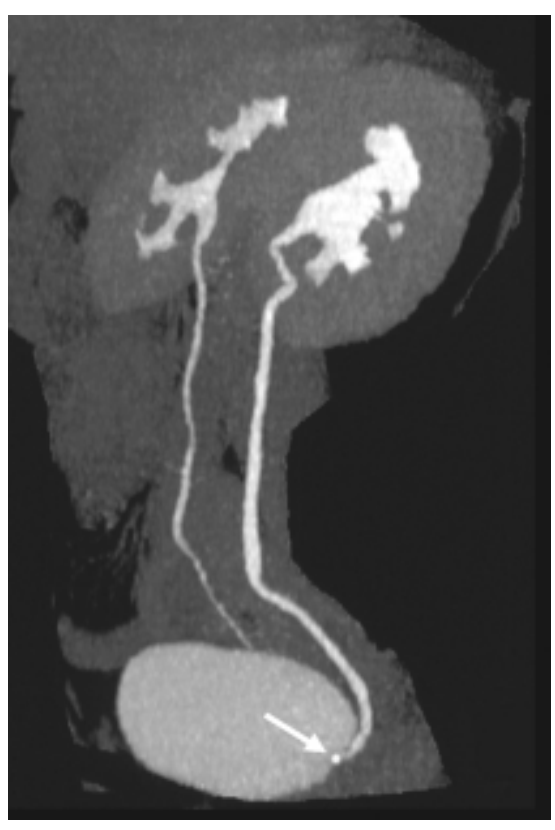

Fig. 3 Forty-threeyear old man with uncharacteristic leftsided flank pain and hematuria. The near lateral MIP image from furosemideenhanced MS-CTU shows a calculus obstructing the left ureterovesical junction. A mild dilatation of the ipsilateral urinary tract is also visible. Multiplanar MIP images confirm that the calculus cannot be misdiagnosed as a phlebolith.

the ureters on MIP images. Calcifications of the iliac arteries were present in some patients but did not simulate ureteral calculi at the site of the uretero-iliac crossover (Fig. 4).

MS-CTU was normal in 10 patients. A total of 16 pathologic findings involving the upper urinary tract were diagnosed in 11 patients: dilatation $(n=4$; Fig. 3$)$, bifid ureter $(n=2$; Fig. 5$)$; 


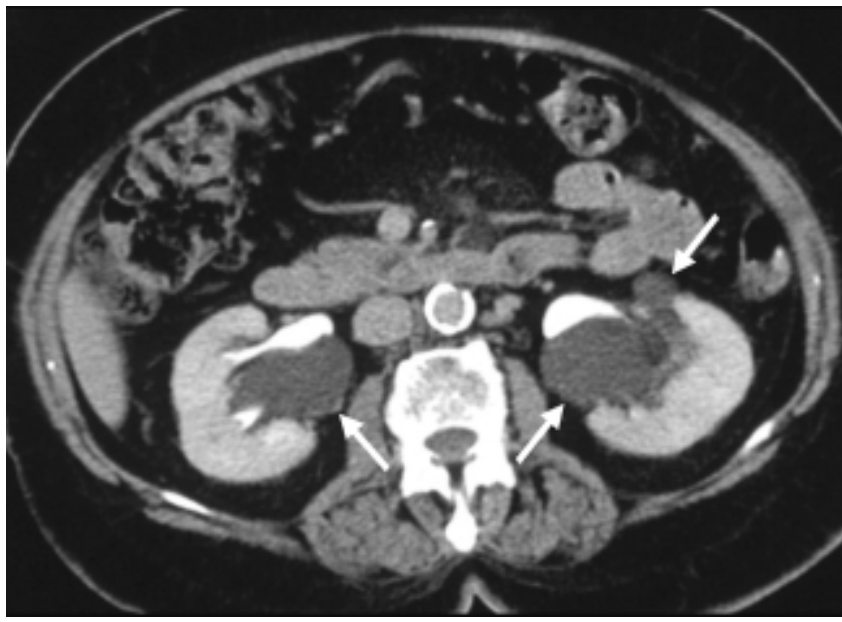

Fig. 4 Sixty-seven-year old woman who sonographically presented with bilateral hydronephrosis. The axial source image from furosemideenhanced MS-CTU (a) discloses the true pathology caused by multiple parapelvic cysts (arrows). The MIP image (b) most obviously demon-

caliceal deformities caused by chronic pyelonephritis $(\mathrm{n}=2$; Fig. 5); multiple parapelvic cysts with displacement of the pelvicaliceal systems $(n=2$; Fig. 4); nephrolithiasis without obstruction $(n=5$; Fig. 2,5), and ureterolithiasis with mild obstruction ( $\mathrm{n}=1$; Fig. 3). On MIP images from furosemideenhanced MS-CTU, calculi could be well differentiated from the adjacent contrast material-enhanced urine (Fig. 2,3,5). Phleboliths were also easily identified and could not be misdiagnosed as ureteral calculi (Fig. 2,3). A transitional cell carcinoma was not found. Extrinsic affection of the ureters was ruled out prior to surgery in the patient with a cervical carcinoma.

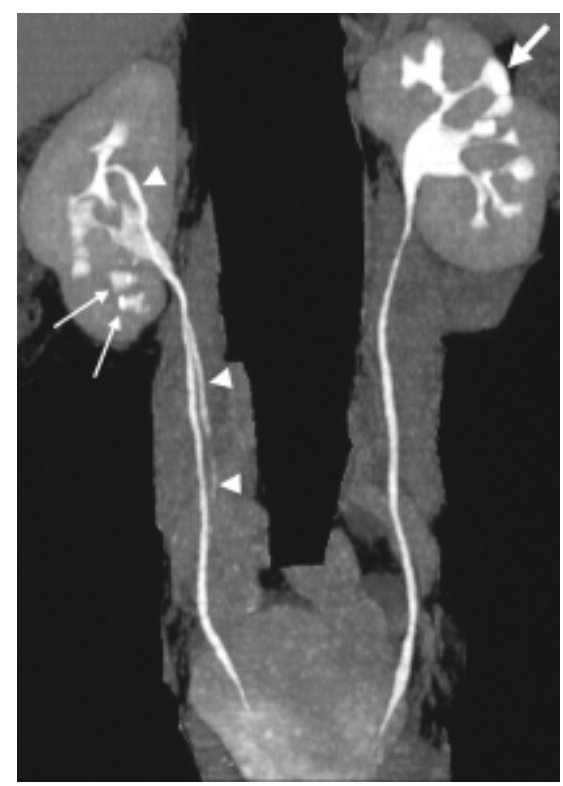

Fig. 5 Marked chronic pyelonephritic changes in a 43year old female patient with a pelvicaliceal duplex system of the right urinary tract. Furosemideenhanced MS-CTU shows distinct bilateral pelvicaliceal deformities including two, previously unknown calculi in the right lower calices (thin arrows) and severe parenchymal atrophy of the left upper pole (thick arrow). The right bifid ureter (arrowheads) is not completely opacified (score $=2)$.

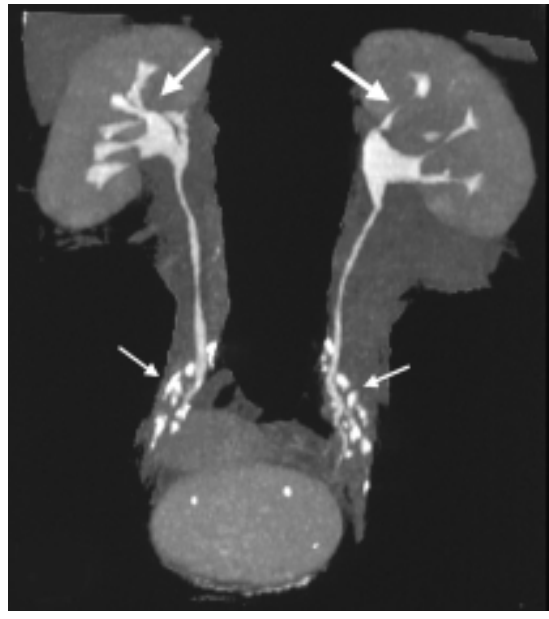

a, b

strates the pelvicaliceal compression and displacement on either side (large arrows). Also note that the ureters are partly superimposed by calcified iliac arteries.

\section{Discussion}

With the development of the multi-slice technology, helical computed tomography has experienced several important advances, which also form the basis for innovations in CT urography. With use of MS-CT, the scan time is markedly shortened by the simultaneous acquisition of, currently, up to four slices, which enables us to carry out a thin-collimation scan protocol of the entire urinary tract in a breath-hold of only 12 to 16 seconds. This aspect does not only help to improve the patients' cooperation during the breath-hold data acquisition. Potentially more important is that MS-CT increases the resolution of the CT data in the axial direction (z-resolution) [4], which especially improves the postprocessing capabilities of the CT data in the manner that we are now able to reconstruct high-quality multiplanar urographic MIP images.

Furthermore, we could demonstrate for MS-CTU that, in analogy to contrast-enhanced MR urography [5], the additional injection of a low-dose diuretic proved to be beneficial for obtaining accurate depiction of the urinary tract morphology. In patients with normal or moderately impaired renal excretory function, the injection of only $10 \mathrm{mg}$ of furosemide constantly achieved a rapid and uniform distribution of the radiopaque contrast material inside the entire upper urinary tract. In contrast to the conventional CTU technique used by McNicholas et al. [3], no additional contrast material injection was necessary using the furosemide-enhanced method. In most of our patients, the ureters were completely visualized although an inconvenient compression device was not used. Low-dose furosemide only led to a mild distension of the nonobstructed urinary tracts. Even fine anatomic details such as caliceal fornices could be visualized on diuretic-enhanced MS-CT urograms. McNicholas et al. reported in their series a partial loss of caliceal detail at conventional window settings due to artefacts from dense contrast material [3]. Such a caliceal „pseudo-blunting“, probably caused by too high papillary and forniceal concentration of contrast material, was not observed with use of low-dose furosemide in our series. Loss of caliceal 
detail was, however, found on our MS-CT urograms obtained using exclusively physiologic saline solution. We could demonstrate that the average radiopacity inside the enhanced pelvicaliceal systems was 4-5 times higher and more inhomogeneous when physiologic saline solution was used instead of furosemide. On the other hand, the furosemide induced dilution of the excreted amount of contrast material and also the increase in urine flow did not destroy the contrast enhancement of the collecting systems, as it may occur in conventional intravenous pyelography. The dilution of the contrast medium also explains for the finding that on MIP images, calculi could still be differentiated inside the opacified urine.

Based on our initial findings obtained from a limited number of patients, we do not claim that the use of low-dose furosemide is the only way to provide high-quality CT urograms. Comparative studies are necessary to find out, if the sole infusion of physiologic saline solution may be as effective as furosemide in achieving the same accurate enhancement of the urinary collecting system. The use of only $10 \mathrm{mg}$ of furosemide is, at least, as simple and harmless as an infusion of $250-500 \mathrm{ml}$ of physiologic saline solution. From our experience, it is advantageous that in patients with normal or moderately impaired excretory function, the injection of low-dose furosemide allows good control over the extent and the timing of the hydration of the urinary tract which cannot be achieved with use of saline solution alone.

In the average patient, an mAs-value of 100 , or probably even less, should provide a sufficient contrast between the opacified urinary tract and the retroperitoneum, which also helps to keep down the radiation dose. Compared to a standard abdominal CT scan, a dose-reduction in CTU is possible because the enhanced urine as a 'high-contrast region-of-interest' allows to reconstruct excellent MIP images even if the source data were acquired at low mAs-values. Moreover, in order to avoid additional radiation exposure to the patient, it is important for the practicability of CTU that the completely opacified urinary tract be imaged by only one acquisition, as far as possible. For this purpose, it proved useful to check the bilateral contrast enhancement of the renal pelvis with a low-dose 'single-slice test image' before starting the definite CTU scan. In our patients, the diuretic-enhanced technique allowed us to complete the examination after a single MS-CTU scan within 10 minutes of contrast material injection. Delayed scans were not necessary in our series. However, delayed scans have to be considered in patients with asymmetrical renal excretion, in whom it will be impossible to get a sufficient urographic overview by acquisition of only a single CT scan.

Another limitation may occur in those applications in which additional oral or rectal administration of contrast material is required. Opacified bowel loops can superimpose parts of the urinary tract and, thus, may interfere with the MIP reconstruction algorithm. Anyway, postprocessing of our large MS-CT data sets, including up to 400 source images, was still time consuming, which was almost exclusively attributed to the limited computing rate of the VOI tool used to eliminate bones, calcified vessels, and bowel loops from MIP images.

Nevertheless, our preliminary results demonstrate that the multi-slice scanning technology and the combined use of contrast material and low-dose furosemide are effective means to improve the feasibility of CT urography in patients who tolerate the application of radiopaque contrast agents. Diureticenhanced MS-CTU allows us to obtain detailed views of the upper urinary tract morphology and together with the diagnostic impact of the axial CT source images, it now appears hard to find any aspect that would favor the preference of standard intravenous pyelography. In the clinical routine, CTU can be easily integrated as part of a multiphase CT protocol of the kidneys, especially, in patients presenting with hematuria of uncertain cause. Because multislice CT provides excellent image resolution, it is allowed to assume that MS-CTU suggests a clinical potential for the detection of early-stage intrinsic tumors inside the non-dilated upper urinary tract. Furthermore, MS-CTU can also be combined with the examination of pelvic organs in patients with gynecologic tumor disease prior to surgical resection. There is no doubt that in acute urolithiasis, unenhanced CT represents the examination of primary choice. MS-CTU can be applied to those diagnostic problem cases, in which unenhanced CT cannot safely answer the question whether a calcified structure is located endoluminally or not. MS-CTU now has reached the state, in which the technique rather competes with MR urography than with conventional pyelography.

\section{Acknowledgement}

The authors thank Roman Iwa for his support in the postprocessing of the CTU data.

\section{References}

${ }^{1}$ Fielding JR, Silverman SG, Rubin GD. Helical CT of the urinary tract. Amer J Roentgenol 1999; 172: 1199-1206

2 Perlman ES, Rosenfield AT, Wexler JS, Glickman MG. CT urography in the evaluation of urinary tract disease. J Comput Assist Tomogr 1996; 20: 620-626

${ }^{3}$ McNicholas MMJ, Raptopoulos VD, Schwartz RK, et al. Excretory phase CT urography for opacification of the urinary collecting system. Amer J Roentgenol 1998; 170: 1261 - 1267

${ }^{4}$ Ohnesorge B, Flohr T, Schaller S, et al. Principles and applications of multi-slice CT. Radiologe 1999; 39: 923-931 [German, English abstract]

${ }^{5}$ Nolte-Ernsting CCA, Bücker A, Adam GB, et al. Gadoliniumenhanced excretory MR urography after low-dose diuretic injection: comparison with conventional excretory urography. Radiology 1998; 209: $147-157$

\section{Nolte-Ernsting M.D.}

Department of Diagnostic Radiology

University Hospital

University of Technology, Aachen

Pauwelsstrasse 30

52057 Aachen

Germany

Tel. ++49 2418088332

Fax ++49 2418888411

E-mail: nolte@rad.rwth-aachen.de 